\title{
Analysis of the center of mass-, rotational- and intramolecular diffusive motions in a monolayer film of intermediate-length alkane molecules adsorbed on a solid surface
}

\author{
F. Y. Hansen* and H. Taub ${ }^{\dagger}$ \\ *Department of Chemistry, Technical University of Denmark, 207-DTU, DK-2800 Lyngby, Denmark. \\ ${ }^{\dagger}$ Department of Physics and Astronomy, University of Missouri-Columbia, Columbia, Missouri 65271, USA.
}

\begin{abstract}
The intramolecular diffusive motions associated with the creation and annihilation of gauche defects in intermediate-length alkane molecules adsorbed on a solid surface are relatively slow and on a time scale of nanoseconds. This has been shown by molecular dynamics (MD) simulations and by high-energy-resolution quasielastic neutron scattering (QNS) on monolayers of tetracosane $\left(n-\mathrm{C}_{24} \mathrm{H}_{50}\right)$ adsorbed on graphite basal-plane surfaces. QNS with the high energy resolution makes it now possible to probe diffusive motions on the same time scale as in nuclear magnetic resonance (NMR) experiments and, in addition, to obtain information about length scales and character of motions through the wavevector dependence of the QNS.
\end{abstract}

\section{INTRODUCTION}

Films of organic molecules on solid surfaces are of great importance in material science and occur in many technological applications such as in adhesion and coatings [1]. Of particular interest are films of $n$-alkanes $\left(\mathrm{C}_{n} \mathrm{H}_{2 n+2}\right)$ and branched alkanes because they are the principal constituents of commercial lubricants [2], and they are also prototypes of more complex polymers.

In the area of biological physics, lipid molecules play an important role as building blocks of the membranes that form the cell walls. The lipid molecules consist of a polar head group to which are attached two alkane chains with typically 14-20 carbon atoms [3]. The phase transition and diffusive motion of the lipid molecules in the membranes are the result of a very delicate balance between the head group and alkane chain properties. By studying the simpler alkane subsystem on a microscopic level, useful information for the understanding of phase transitions and molecular diffusion in the much more complicated bilayer lipid membranes may be obtained.

Previously, we have conducted extensive molecular dynamics (MD) simulations of the structure, melting transition, and diffusive motion in monolayer films of tetracosane $(n=24)$ and dotriacontane $(n=32)$ molecules adsorbed on the basal-planes of graphite [4]. For our neutron scattering experiments, we have chosen a largesurface-area substrate of exfoliated graphite. At low temperature $(<215 \mathrm{~K})$, the adsorbed molecules form a $2 \mathrm{D}$ crystalline structure which is transformed at $215 \mathrm{~K}$ into a smectic crystalline phase that melts at about 340-350 $\mathrm{K}$. The simulated structures and melting transitions have been compared with the results of neutron diffraction measurements, while the diffusive motions inferred from the simulations have been used to interpret the results of QNS experiments.

The low-energy conformation of an alkane molecule is one where all dihedral bonds (the $\mathrm{C}-\mathrm{C}$ bonds along the carbon backbone of the molecule) are in the transconformation. The potential energy function for rotation around the dihedral bonds also has a local minimum at approximately 120 degrees from the trans conformation, and this conformation is usually referred to as a gauche conformation or a gauche defect. One of the predictions of the MD simulations [4] was that the lattice melting of the ordered low-temperature structure was driven by a chain melting. That is, well below the melting temperature (340 K for tetracosane (C24) and $350 \mathrm{~K}$ for dotriacontane (C32) monolayers), gauche defects are confined to the ends of the otherwise all-trans carbon backbone of the molecules. However, beginning at about $10 \mathrm{~K}$ below the melting temperature, gauche defects penetrate into the central region of the molecules. When their number reaches a certain level, a simultaneous chain melting and lattice melting occurs. This type of melting has also been observed in the gel-to-fluid transition of bilayer lipid membranes by NMR $[3,4]$.

Our MD simulations have shown that the diffusive 
motions associated with the creation and annihilation of gauche defects are relatively slow and on the time scale of nanoseconds. We have now been able to corroborate the MD predictions by using QNS measurements taken on the High Flux Backscattering Spectrometer (HFBS) at the Center for Neutron Research, National Institute of Standards and Technology (NIST) [5]. The instrument has a very high energy resolution of $\sim 1 \mu \mathrm{eV}$ allowing us to probe motions on the nanosecond time scale as well as a sufficient throughput to detect scattering from a molecular monolayer adsorbed on an exfoliated graphite substrate. This is the same time scale that can be probed by NMR; but, in addition, the wave vector dependence of the QNS gives information about length scales in the systems and may be used in the analysis of the types of motions involved.

In this paper, we describe a method for projecting out the translational, rotational, and intramolecular diffusive motions of a molecule. This allows us to determine the signature of the various kinds of molecular diffusive motion in a QNS experiment and compare the results directly with experiments. The time scales of these motions are often quite different and, by choice of a spectrometer with the proper energy resolution, it may be possible to study each of these motions separately. Details of the experiments at the new HFBS at NIST are given in the paper by Taub et al. [6].

\section{TIME CORRELATION FUNCTIONS IN NEUTRON SCATTERING}

In a neutron scattering experiment, we distinguish between coherent and incoherent scattering where the intensity of the former is proportional to the coherent scattering function $S_{\mathrm{coh}}(\mathbf{Q}, \omega)$ and the latter to the incoherent scattering function $S_{\text {inc }}(\mathbf{Q}, \omega)$ [7]. $\mathbf{Q}$ is the wavevector transfer and $\hbar \omega$ the energy change of the scattered neutrons. For a fluid and a powder, the scattering functions only depend on the magnitude $|\mathbf{Q}|$ of the wavevector which will be the case here. The scattering functions reflect the dynamics of the system and may be calculated in an MD simulation. For $\hbar \omega=0$, we have elastic scattering. Of particular interest here are the scattering functions for $\hbar \omega \rightarrow 0$, usually referred to as QNS, and the motions associated with that scattering are referred to as diffusive, since they are not oscillatory in this limit. Examples are translational and rotational diffusive motions.

All experiments are done on protonated alkane molecules. Since the hydrogen atom has a very large incoherent scattering cross section compared to the coherent cross sections for both hydrogen and carbon atoms, the scattering will be strongly dominated by the incoherent scattering. We shall therefore only be inter- ested in the incoherent scattering function $S_{\text {inc }}(Q, \omega)$. It is the time Fourier transform of the intermediate self-scattering function $F_{\mathrm{s}}(Q, t)$

$$
S_{\text {inc }}(Q, \omega)=\frac{1}{2 \pi} \int_{-\infty}^{\infty} d t \exp (-i \omega t) F_{\mathrm{s}}(Q, t)
$$

that is given by the expression

$$
F_{s}(\mathbf{Q}, t)=\frac{1}{N} \sum_{j=1}^{N}\left\langle\exp \left(i \mathbf{Q} \cdot\left(\mathbf{r}_{j}(t)-\mathbf{r}_{j}(0)\right)\right)>.\right.
$$

This function is readily calculable from a time series of atomic positions $\mathbf{r}_{j}(t)$ generated in a MD simulation. The sum extends over all $N$ atoms in the system, and the bracket $\langle\cdots\rangle$ indicates an ensemble average. The simulations have been done by canonical sampling as described in an earlier publication [4].

Let us rewrite the intermediate scattering function in the following way

$$
\begin{aligned}
& F_{S}(Q, t)=F_{S}(Q, \infty)+F_{s}^{\prime}(Q, t) \text { with } \\
& F_{S}^{\prime}(Q, t) \rightarrow 0 \text { for } t \rightarrow \infty .
\end{aligned}
$$

Then the scattering function will be

$$
S_{\mathrm{inc}}(Q, \omega)=2 \pi F_{s}(Q, \infty) \delta(\omega)+S_{\mathrm{inc}}^{\prime}(Q, \omega) .
$$

This shows that the incoherent scattering function, and hence the incoherent scattering intensity, may consist of two contributions. The first term on the right hand side gives an elastic contribution when $F_{S}(Q, \infty)$ is different than zero, that is, when the atoms do not move far away from their original positions as in rotational or vibrational motion. When the atoms move far away, there is no elastic contribution, since $F_{s}(Q, \infty)$ will be zero. The second term in the expression gives the frequency dependent part of the scattering function and reflects the kind of diffusive motion involved.

\section{ANALYSIS OF THE MOLECULAR MOTIONS}

Any motion of the atoms in a molecule may be described as a combination of a center of mass motion, a rotational motion, and an intramolecular motion. The time scales of these motions are often different, and it will be useful to have a method that allows us to study the various types of motion in an MD simulation. Of particular interest here, the intramolecular motion.

Let us call the position vector of atom $i$ in molecule $m$ at time $t \mathbf{r}_{i m}(t)$. Then the center of mass position vector is given by the standard expression [8]

$$
\mathbf{R}_{m}(t)=\frac{\sum_{i=1}^{M} m_{i} \mathbf{r}_{i m}(t)}{\sum_{i=1}^{M} m_{i}}
$$


The rotational motion of a molecule may be determined in the following way. At time $t_{1}$, let the three principal axes of rotation be $\mathbf{e}_{1}\left(t_{1}\right), \mathbf{e}_{2}\left(t_{1}\right)$ and $\mathbf{e}_{3}\left(t_{1}\right)$, such that

$$
\left[\mathbf{e}_{1}\left(t_{1}\right), \mathbf{e}_{2}\left(t_{1}\right), \mathbf{e}_{3}\left(t_{1}\right)\right]=[\mathbf{i}, \mathbf{j}, \mathbf{k}]\left[E\left(t_{1}\right)\right] .
$$

$\mathbf{i}, \mathbf{j}, \mathbf{k}$ are the basis vectors for the laboratory-fixed cartesian coordinate system. The elements of the $i t h$ column of the $(3 \times 3)$ matrix $E\left(t_{1}\right)$ are the cartesian coordinates of the $i t h$ principal axis $\mathbf{e}_{i}\left(t_{1}\right)$; they are determined by a diagonalization of the inertia tensor for the molecule at time $t_{1}[8]$. We may invert Eq. (6) and find

$$
\left[\mathbf{e}_{1}\left(t_{1}\right), \mathbf{e}_{2}\left(t_{1}\right), \mathbf{e}_{3}\left(t_{1}\right)\right]\left[E\left(t_{1}\right)\right]^{-1}=[\mathbf{i}, \mathbf{j}, \mathbf{k}] .
$$

At some later time $t_{2}$, the three principal axes of rotation are $\mathbf{e}_{1}\left(t_{2}\right), \mathbf{e}_{2}\left(t_{2}\right)$ and $\mathbf{e}_{3}\left(t_{2}\right)$, that is

$$
\left[\mathbf{e}_{1}\left(t_{2}\right), \mathbf{e}_{2}\left(t_{2}\right), \mathbf{e}_{3}\left(t_{2}\right)\right]=[\mathbf{i}, \mathbf{j}, \mathbf{k}]\left[E\left(t_{2}\right)\right] .
$$

Before we can project out the rigid rotational displacement of a given atom in going from time $t_{1}$ to time $t_{2}$, we need to determine the atomic position vectors with respect to the center of mass coordinate $\mathbf{R}_{m}\left(t_{1}\right)$ of molecule $m$. Let us call this "relative" atomic position vector $\mathbf{s}_{i m}\left(t_{1}\right)$, then

$$
\mathbf{s}_{i m}\left(t_{1}\right)=\mathbf{r}_{i m}\left(t_{1}\right)-\mathbf{R}_{m}\left(t_{\mathrm{l}}\right)
$$

The "relative" position vector $\mathbf{s}_{i m}\left(t_{1}\right)$ may be written in terms of the cartesian components arranged in the $(3 \times 1)$ column vector $\left[s_{i m}\left(t_{1}\right)\right]$ like

$$
\mathbf{s}_{i m}\left(t_{1}\right)=[\mathbf{i}, \mathbf{j}, \mathbf{k}]\left[s_{i m}\left(t_{1}\right)\right]=[\mathbf{i}, \mathbf{j}, \mathbf{k}]\left[r_{i m}\left(t_{1}\right)-R_{m}\left(t_{1}\right)\right]
$$

and from Eq. (7), in the basis of $\mathbf{e}_{1}\left(t_{1}\right), \mathbf{e}_{2}\left(t_{1}\right), \mathbf{e}_{3}\left(t_{1}\right)$

$$
\mathbf{s}_{i m}\left(t_{1}\right)=\left[\mathbf{e}_{1}\left(t_{1}\right), \mathbf{e}_{2}\left(t_{1}\right), \mathbf{e}_{3}\left(t_{1}\right)\right]\left[E\left(t_{1}\right)\right]^{-1}\left[s_{i m}\left(t_{1}\right)\right] .
$$

If the displacement of the ith atom in molecule $m$ from time $t_{1}$ to time $t_{2}$ is given by a rigid rotation of the molecule, as defined by the rotation of the principal axes of rotation, then the coordinates of atom $i$ in the $\mathbf{e}_{1}\left(t_{2}\right), \mathbf{e}_{2}\left(t_{2}\right), \mathbf{e}_{3}\left(t_{2}\right)$ system should be the same as in the $\mathbf{e}_{1}\left(t_{1}\right), \mathbf{e}_{2}\left(t_{1}\right), \mathbf{e}_{3}\left(t_{1}\right)$ system, that is

$$
\begin{aligned}
\mathbf{s}_{i m}^{r}\left(t_{2}\right) & =\left[\mathbf{e}_{1}\left(t_{2}\right), \mathbf{e}_{2}\left(t_{2}\right), \mathbf{e}_{3}\left(t_{2}\right)\right]\left[E\left(t_{1}\right)\right]^{-1}\left[s_{i m}\left(t_{1}\right)\right] \\
& =[\mathbf{i}, \mathbf{j}, \mathbf{k}]\left[E\left(t_{2}\right)\right]\left[E\left(t_{1}\right)\right]^{-1}\left[s_{i m}\left(t_{1}\right)\right] \\
& \equiv[\mathbf{i}, \mathbf{j}, \mathbf{k}] \operatorname{Rot}_{m}\left(t_{2}, t_{1}\right)\left[s_{i m}\left(t_{1}\right)\right]
\end{aligned}
$$

We have used Eq. (8), and $\mathbf{s}_{i m}^{r}\left(t_{2}\right)$ is the position vector of atom $i$ in molecule $m$ relative to the center of mass position at time $t_{2}$, if the atom has performed a rigid rotational motion from time $t_{1}$ to time $t_{2} \cdot \operatorname{Rot}_{m}\left(t_{2}, t_{1}\right)$ is a (3x3) matrix operator given by

$$
\operatorname{Rot}_{m}\left(t_{2}, t_{1}\right)=\left[E\left(t_{2}\right)\right]\left[E\left(t_{1}\right)\right]^{-1}
$$

which may be used to determine the "relative" position vector of atom $i$, when it performs a rigid rotation from time $t_{1}$ to time $t_{2}$ around the center of mass.

The MD simulation generates the atomic positions at time $t_{2}$. From those we may determine the center of mass position at time $t_{2}$ using Eq. (5), and the "relative" atomic positions $\boldsymbol{s}_{i m}\left(t_{2}\right)$ at that time are given by

$$
\begin{aligned}
\mathbf{s}_{i m}\left(t_{2}\right) & =\mathbf{r}_{i m}\left(t_{2}\right)-\mathbf{R}_{m}\left(t_{2}\right) \\
& =\mathbf{s}_{i m}^{r}\left(t_{2}\right)+\mathbf{s}_{i m}^{i n t}\left(t_{2}\right)
\end{aligned}
$$

Since $\mathrm{s}_{i m}\left(t_{2}\right)$ is known from the MD simulation and $\mathbf{s}_{i m}^{r}\left(t_{2}\right)$ may be determined from Eq. (12), the intramolecular displacement of atom $i$ in molecule $m$ from $t_{1}$ to $t_{2}$, $\mathbf{s}_{i m}^{i n t}\left(t_{2}\right)$, is given by this equation.

\section{RESULTS AND DISCUSSION}

The energy resolution and dynamical range in the MD simulation were chosen to match those in the experiment. The energy resolution is given as $1 / \tau_{\max }\left(s^{-1}\right)$ where $\tau_{\max }(\mathrm{s})$ is the length of the time series in the MD simulation, and the dynamic range $1 /(2 d \tau)\left(s^{-1}\right)$ is determined by the time $d \tau$ (s) between two consecutive recordings of the atomic positions in the time series.

It is clear from the definition in Eq. (1) that the intermediate scattering function $F_{s}(Q, t)$ is equal to unity at $t=0$. As discussed previously, it decays either to a nonzero constant level or to zero at long times, depending on the character of the motion. As an example, let us look at the intermediate scattering function in Fig. 1 for the rotational and intramolecular motions of C24 molecules in a monolayer. We note that the intermediate scattering functions level off at a non-zero level at long times. This implies that there also will be elastic scattering from these modes. In Fig. 1(a) we note that the intermediate scattering function for both modes has dropped directly to the long-time level at the first time step after the initial time for both modes. This means that the modes are too fast on the time scale chosen in the simulations for us to follow the decay. In order to do so we need to choose a smaller $d \tau$. The functions may be approximated by a delta function $\delta(t)$ at time zero and by a constant at any other time. Since the scattering function $S_{\text {inc }}(Q, \omega)$ is the Fourier transform of the intermediate scattering function, Eq.(1), the delta function will produce a constant intensity, independent of $\omega$, and the constant time-independent part an elastic scattering at $\omega=0$.

It was found that the translational mode also was too fast at about $260 \mathrm{~K}$ with the given time resolution, so the scattered intensity will consist of only an elastic part and a featureless constant quasielastic scattering. Since the modes will be faster with increasing temperature, we would not expect to find any other quasielastic scatter- 


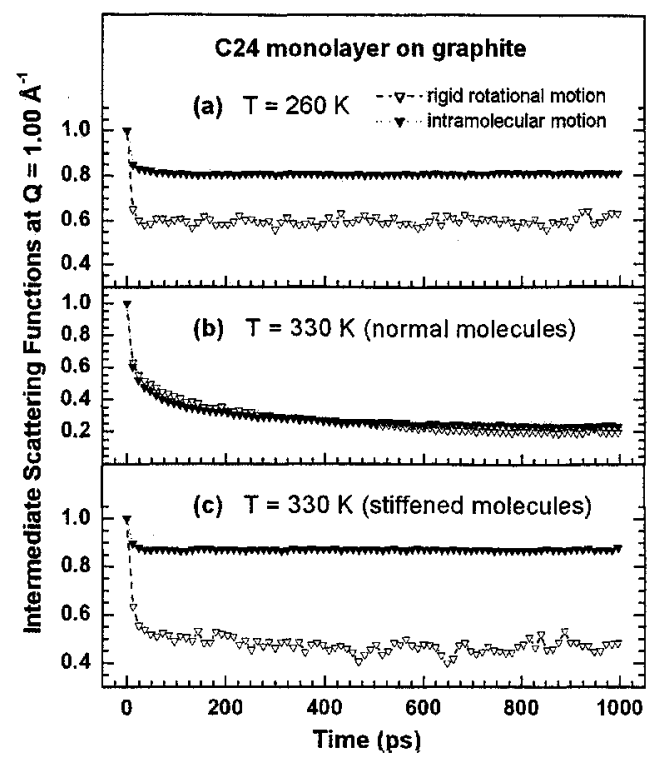

FIGURE 1. Intermediate scattering functions for the rotational- and intramolecular motions of $\mathrm{C} 24$ molecules.

ing within the dynamic range of the HFBS unless some new slow modes were activated. This happened at about $330 \mathrm{~K}$ as can be concluded from Fig. 1(b). Contrary to what we saw in Fig. 1(a), it is now possible to follow the decay of both the rotational and intramolecular modes for several hundred picoseconds before they reach a constant non-zero plateau. Our MD simulations of the melting transition [4] had shown that gauche defects started to penetrate into the central region of the molecules at about $330 \mathrm{~K}, 10 \mathrm{~K}$ below the melting temperature. The appearance of the new slow modes was therefore interpreted as originating from the motion associated with the creation and annihilation of the defects. The slow-down of the rotational motions also is explained by the change in shape of the molecules, when gauche defects are introduced. This leads to a larger moment of inertia for the rotation around the long axis that gives the primary contribution to the scattering from the rotational diffusive motion. To substantiate this conclusion, we stiffened the molecules around the dihedral torsion angles to prevent any formation of gauche defects and found that the slow modes disappeared as is evident from Fig. 1(c).

The half-width at half-maximum (HWHM) of the scattering function $S_{\mathrm{inc}}(Q, \omega)$ for both experiments and simulation has been plotted in Fig. 2(a) as a function of $Q$. We note that the experimental results at $285 \mathrm{~K}$ have

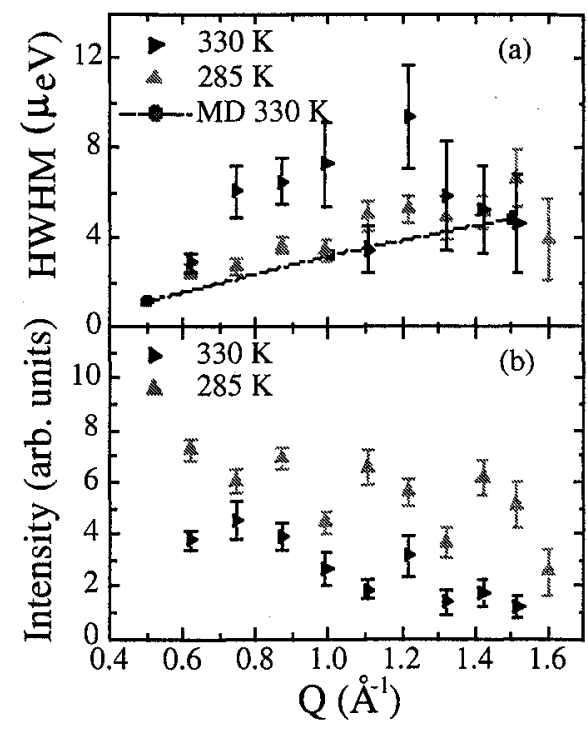

FIGURE 2. Half-width at half-maximum (HWHM) of the scattering function and scattered intensity for a monolayer of C24 molecules.

no counterpart in the simulation, because all modes were found to be outside the dynamical range of the HFBS at that temperature. The reason is that in the united atom model used in the simulations the moment of inertia for rotation about the long molecular axis is about a factor of two too small. This makes the rotational motion faster than in the experiments.

At $330 \mathrm{~K}$, on the other hand, we find qualitative agreement of the HWHM of the QNS between experiments and the simulations and interpret this as an experimental verification of the melting mechanism proposed in Ref. [4]. In addition, our simulations show a $Q$-dependence of the QNS intensity similar to that measured [see Fig. 2(b)]. We conclude that our results demonstrate the potential of QNS for investigating slow modes on a nanosecond time scale in samples as small as a molecular monolayer.

\section{REFERENCES}

1. Xia, T.K. et al., Phys. Rev. Lett. 69, 1967-1970, (1992).

2. Xia, T.K. et al., Science 261, 1310-1312, (1993).

3. Small, D.M. The Physical Chemistry of Lipids, Plenum, New York, 1986, pp. 1-19.

4. Hansen, F.Y. et al., Phys. Rev. Lett. 83, 2362-2365, (1999).

5. Meyer, A. et al., Rev. Sci. Instrum. 74, 2759-2777, (2003).

6. Taub, H. et al., this proceedings, 2003.

7. Marshall, W. and Lovesey, S.W., Theory of Thermal Neutron Scattering, Oxford University Press 1971, Oxford, pp. 38-45, 370-383.

8. Goldstein, H. Classical Mechanics, Addison-Wesley Publishing Company, Reading 1980, pp. 188-203. 